\title{
The Alternating Access Transport Mechanism in LacY
}

\author{
H. Ronald Kaback • Irina Smirnova • \\ Vladimir Kasho $\cdot$ Yiling Nie $\cdot$ Yonggang Zhou
}

Received: 23 September 2010/ Accepted: 5 November 2010/Published online: 16 December 2010

(c) The Author(s) 2010. This article is published with open access at Springerlink.com

\begin{abstract}
Lactose permease of Escherichia coli (LacY) is highly dynamic, and sugar binding causes closing of a large inward-facing cavity with opening of a wide outward-facing hydrophilic cavity. Therefore, lactose/ $\mathrm{H}^{+}$symport via LacY very likely involves a global conformational change that allows alternating access of single sugar- and $\mathrm{H}^{+}$-binding sites to either side of the membrane. Here, in honor of Stephan H. White's seventieth birthday, we review in camera the various biochemical/biophysical approaches that provide experimental evidence for the alternating access mechanism.
\end{abstract}

Keywords Lactose $\cdot$ Permease $\cdot$ Symport $\cdot$ Transport $\cdot$ Membrane $\cdot$ Membrane protein

H. R. Kaback · I. Smirnova · V. Kasho - Y. Nie · Y. Zhou Department of Physiology and Department of Microbiology, University of California Los Angeles, Los Angeles, CA 90095, USA

H. R. Kaback

Immunology and Molecular Genetics, University of California Los Angeles, Los Angeles, CA 90095, USA

H. R. Kaback

Molecular Biology Institute, University of California

Los Angeles, Los Angeles, CA 90095, USA

H. R. Kaback ( $\square)$

MacDonald Research Laboratories (Rm 6720),

UCLA, 675 Charles E. Young Drive South,

Los Angeles, CA 90095-1662, USA

e-mail: rkaback@mednet.ucla.edu

\section{Introduction}

The lactose permease of Escherichia coli (LacY), which catalyzes the coupled symport of a galactopyranoside and an $\mathrm{H}^{+}$, is a paradigm for the major facilitator superfamily (MFS) of membrane transport proteins. LacY has been solubilized, purified and reconstituted into proteoliposomes in a fully functional state (reviewed in Viitanen et al. 1986). Furthermore, X-ray crystal structures of the conformationally restricted mutant Cys $154 \rightarrow$ Gly have been solved in an inward-facing conformation (Abramson et al. 2003; Mirza et al. 2006), and the crystal structure of wildtype LacY exhibits the same conformation (Guan and Kaback 2006; Guan et al. 2007). Both structures have 12 transmembrane $\alpha$-helices, most of which are shaped irregularly, organized into two pseudosymmetrical sixhelix bundles surrounding a large interior hydrophilic cavity open to the cytoplasm only (Fig. 1). The sugarbinding site and the residues involved in $\mathrm{H}^{+}$translocation are at the approximate middle of the molecule at the apex of the hydrophilic cavity and distributed so that the side chains important for sugar recognition are predominantly in the N-terminal helix bundle and the side chains that form an $\mathrm{H}^{+}$-binding site are mainly in the $\mathrm{C}$-terminal bundle (Smirnova et al. 2009b). The periplasmic side of LacY is tightly packed, and the sugar-binding site is inaccessible from that side of the molecule. A similar structure has been observed for the X-ray structure of GlpT, which has little or no sequence homology with LacY and catalyzes exchange of inorganic phosphate for glycerol-3-P across the membrane (Huang et al. 2003).

Wild-type LacY is highly dynamic. H/D exchange of backbone amide protons in wild-type LacY occurs at rapid rate (le Coutre et al. 1998; Patzlaff et al. 1998; Sayeed and Baenziger 2009), and sugar binding by wild-type LacY is 


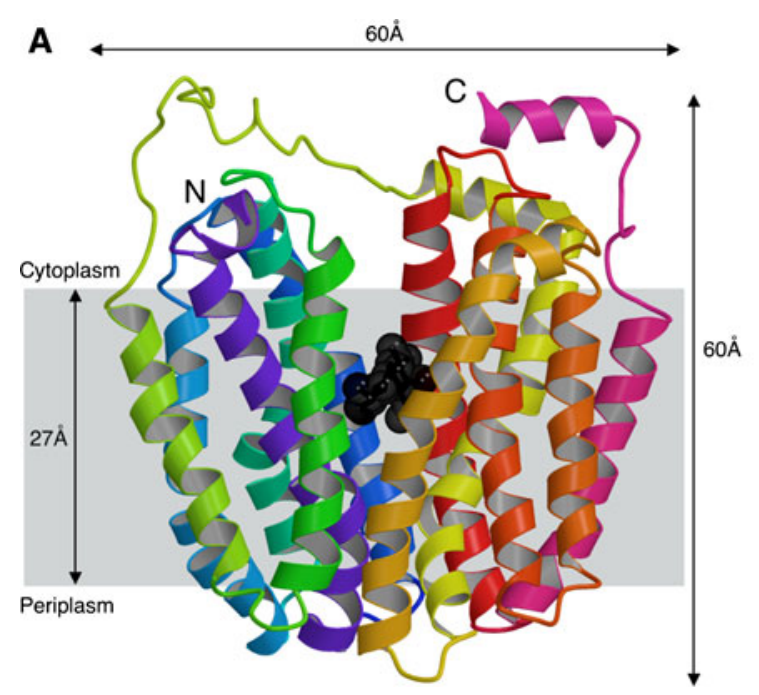

B

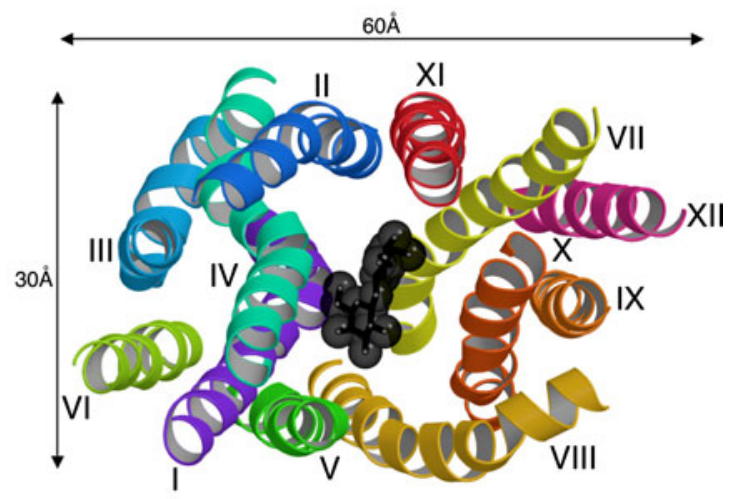

Fig. 1 Overall structure of LacY. a Ribbon representation of LacY viewed parallel to the membrane. b Ribbon representation of LacY viewed along the membrane normal from the cytoplasmic side. TDG is represented as black spheres

mostly entropic (Nie et al. 2006), inducing widespread conformational changes (reviewed in Guan and Kaback 2006; Kaback 2005; Kaback et al. 2001). More specifically, site-directed alkylation (reviewed in Kaback et al. 2007; Nie et al. 2007, 2008; Nie and Kaback 2010), single molecule fluorescence resonance energy transfer (Majumdar et al. 2007), double electron-electron resonance (Smirnova et al. 2007), site-directed cross-linking (Zhou et al. 2008) and Trp-quenching studies (Smirnova et al. 2009a) each provide independent evidence that sugar binding increases the open probability of a wide hydrophilic cleft on the periplasmic side of LacY with closing of the cytoplasmic cavity so that the sugar- and $\mathrm{H}^{+}$-binding sites become alternatively accessible to either side of the membrane (the alternating access model). It has also been shown that the periplasmic cleft must close, as well as open, for translocation of sugar across the membrane to occur (Liu et al. 2010; Zhou et al. 2008, 2009).

Notably, in the conformationally restricted mutant C154G LacY, sugar binding is enthalpic and the periplasmic cleft is paralyzed in an open conformation (Majumdar et al. 2007; Nie et al. 2008; Smirnova et al. 2007). However, all X-ray structures of LacY (C154G as well as wildtype LacY) exhibit the same inward-facing conformation. Therefore, it is likely that the crystallization process selects a single conformer of LacY that is in the lowest free-energy state.

A functional LacY molecule devoid of its eight native Cys residues ( $\mathrm{C}$-less $\mathrm{LacY}$ ) has been engineered by constructing a cassette $l a c Y$ gene with unique restriction sites about every 100 bp (van Iwaarden et al. 1991). Utilizing this cassette $l a c Y$ for Cys-scanning mutagenesis, a highly useful library of molecules with a single-Cys residue at virtually every position of LacY has been constructed (Frillingos and Kaback 1996). Cys is average in bulk, relatively hydrophobic and amenable to highly specific modification. Therefore, Cys-scanning mutagenesis has been used in combination with biochemical and biophysical techniques to reveal membrane topology, accessibility of intramembrane positions to the aqueous or lipid phase of the membrane and spatial proximity between transmembrane domains.

Here, the experimental approaches that provide a strong case for the alternating access model are reviewed cursorily.

\section{Site-Directed Alkylation}

Site-directed alkylation (SDA) of sylfhydryl thiols by radiolabeled $N$-ethylmaleimide (NEM) or fluorescent tetramethylrhodamine-5-maleimide (TMRM), which are membrane-permeant alkylating agents, has been used to study the reactivity of single-Cys LacY mutants in the $\mathrm{C}$-less background in right-side-out (RSO) membrane vesicles. The approach provides important information about the structure, function and dynamics of LacY (reviewed in Guan and Kaback 2007). The reactivity/accessibility of Cys residues depend on the surrounding environment and are limited by close contacts between transmembrane helices and/or the low dielectric of the environment. Ligand binding increases NEM reactivity of single-Cys replacements located predominantly on the periplasmic side of LacY and decreases reactivity of those located predominantly on the cytoplasmic side (Guan and Kaback 2007). The pattern suggests that during sugar transport a periplasmic pathway opens with closing of the inward-facing cavity so that the 


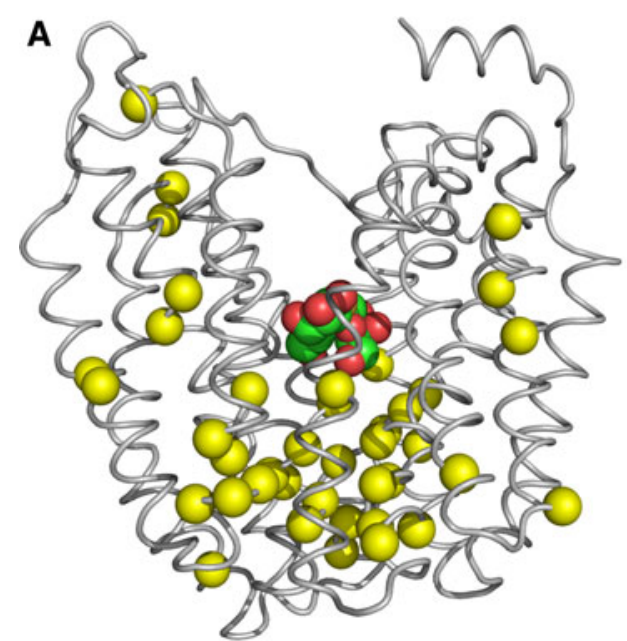

Fig. 2 Distribution of Cys replacements that exhibit changes in reactivity with NEM in the presence of TDG. a Positions of Cys replacements that exhibit a significant increase in reactivity with NEM (gold spheres) superimposed on the backbone of LacY viewed perpendicular to the plane of the membrane. TDG is shown as a CPK model at the apex of the inward-facing cavity. b Positions of Cys

sugar-binding site is alternatively accessible to either side of the membrane (Fig. 2).

Most recently, the simple, more facile alkylation method with TMRM (Nie et al. 2007, 2008, 2009) was utilized to examine the effect of sugar binding on alkylation of singleCys LacY mutants either in RSO membrane vesicles or with purified proteins in dodecyl- $\beta$, D-maltopyranoside (DDM) micelles (Nie and Kaback 2010). Experiments were carried out at $0^{\circ} \mathrm{C}$, where thermal motion is restricted (Venkatesan and Kaback 1998; Venkatesan et al. 2000a, 2000b), and linear rates of labeling were readily obtained (Fig. 3). TMRM labeling is almost negligible, with LacY containing each of five single-Cys residues at positions on the periplasmic side of the sugar-binding site in RSO membrane vesicles or with purified protein in DDM micelles. Therefore, each of these single-Cys replacements is unreactive and/or inaccessible to the alkylating agent. The observations are consistent with the interpretation that $\mathrm{LacY}$ in the native bacterial membrane is in a conformation similar to that of the X-ray crystal structures in the absence of ligand. The periplasmic side is tightly closed, and an open cavity is present facing the cytoplasm (the inwardfacing conformation) (Abramson et al. 2003; Guan et al. 2007; Mirza et al. 2006).

As postulated by the alternating access model, on the cytoplasmic side of the sugar-binding site, each of five singleCys replacement mutants labels at a rapid rate in the absence of sugar both in RSO membrane vesicles and with purified protein in DDM. Moreover, the tight-binding lactose homologue $\beta$-D-galactopyranosyl 1-thio- $\beta$, D-galactopyranoside

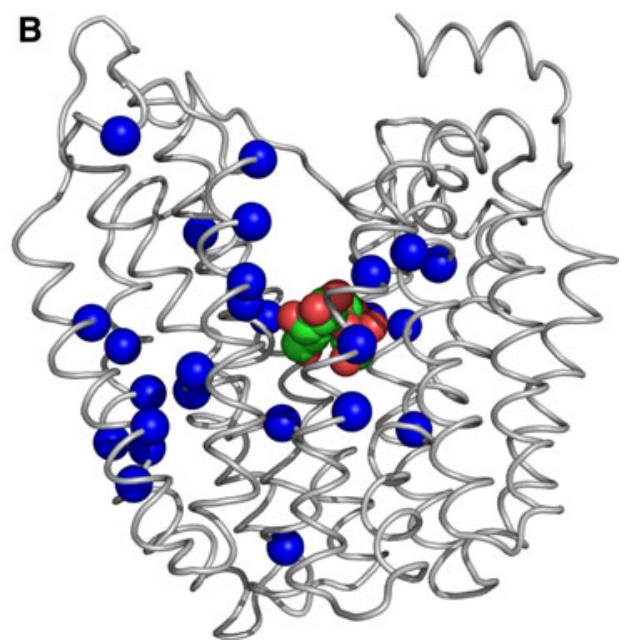

replacements that exhibit a significant decrease in reactivity with NEM (blue spheres) superimposed on the backbone of LacY viewed perpendicular to the plane of the membrane. The cytoplasmic surface is at the top, and TDG is shown as a CPK model at the apex of the inward-facing cavity (Color figure online)

(TDG) decreases the rate of TMRM labeling either in the membrane or with purified protein in DDM (Fig. 3; Table 1). The findings agree with a variety of other measurements (see below) showing that sugar binding induces closing of the cytoplasmic cavity and reduced reactivity/accessibility to alkylating agents.

The average increase in periplasmic TMRM labeling observed in the presence of TDG in RSO vesicles is $\sim 10$-fold, and the average cytoplasmic decrease in the presence of TDG is very similar (approximately ninefold) (Table 1). With purified single-Cys proteins in DDM, the comparable averages are approximately sixfold and approximately fivefold. Thus, the change in TMRM labeling induced by sugar on opposite faces of LacY appears to be about the same in RSO vesicles or with the purified single-Cys mutants in DDM. Therefore, the data provide further evidence not only that sugar binding markedly increases the open probability on the periplasmic side but that sugar binding also increases the probability of closing on the inside, the implication being that opening and closing may be reciprocal. However, reciprocity may not be obligatory as evidence has been presented showing that the periplasmic pathway is fixed in an open conformation in the $\mathrm{C} 154 \mathrm{G}$ mutant, while the cytoplasmic cavity is able to close and open (Majumdar et al. 2007; Nie et al. 2008; Smirnova et al. 2007). It has also been demonstrated (Liu et al. 2010) that replacement of Asp68 with Glu at the cytoplasmic end of helix II blocks sugar-induced opening of the periplasmic cleft but has little or no effect on closing of the cytoplasmic cavity. 

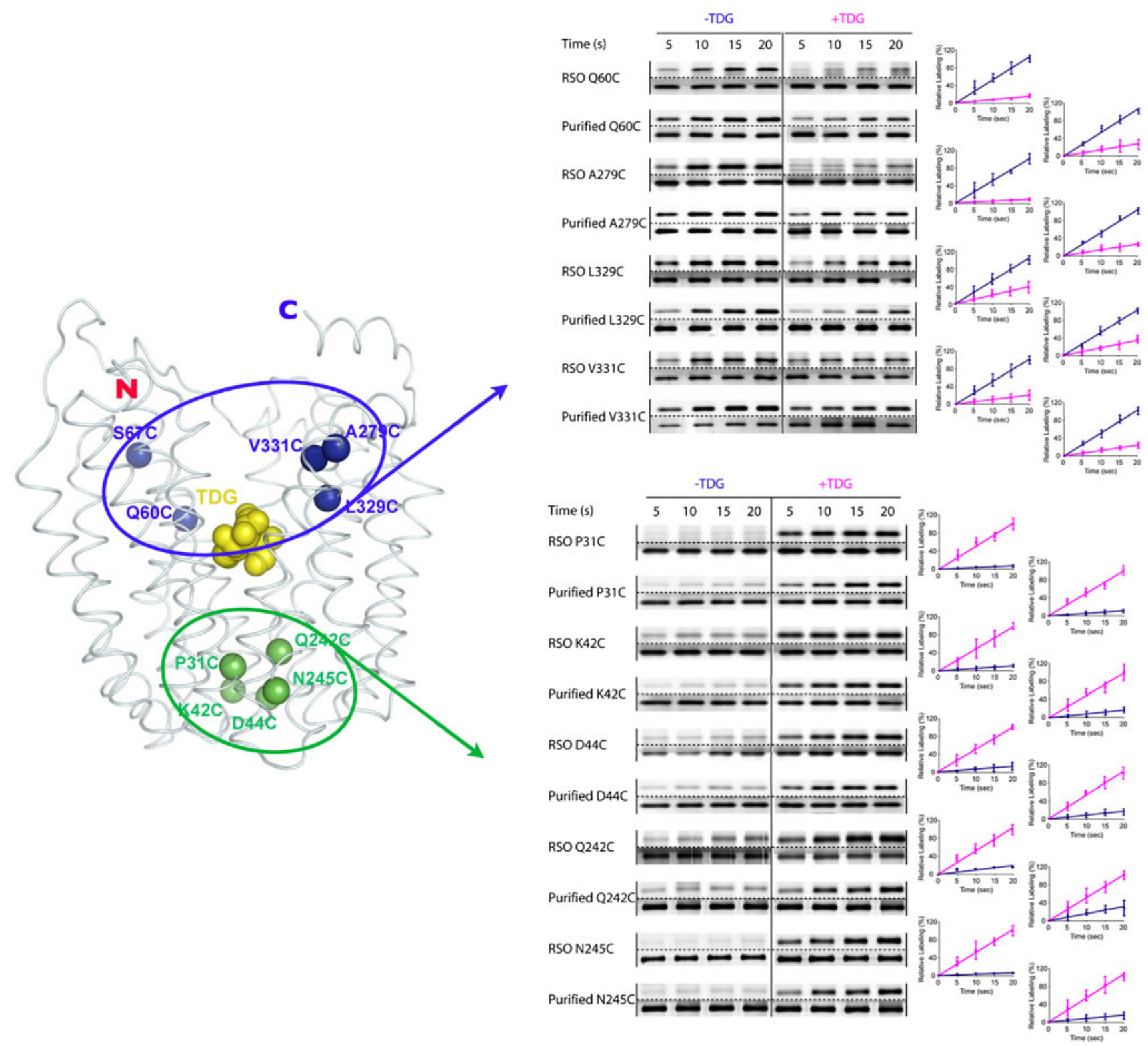

Fig. 3 TMRM labeling of cytoplasmic (top) or periplasmic (bottom) single-Cys mutants in RSO membrane vesicles or as purified proteins in DDM. Labeling of cytoplasmic single-Cys LacY mutants Q60C, S67C, A279C, L329C and V331C or periplasmic single-Cys LacY mutants Q31C, K42C, D44C, Q242C and N245C was performed with $40 \mu \mathrm{M}$ TMRM (RSO membrane vesicles) or $4 \mu \mathrm{M}$ TMRM (with purified proteins in DDM) for given times at $0^{\circ} \mathrm{C}$ in the absence of

Although the sugar-induced changes in the global conformation of LacY are qualitatively similar in RSO membrane vesicles and with the purified mutants in DDM, it is notable that the magnitude of the effects is somewhat smaller with the purified mutant proteins (Table 1). Thus, the increases and decreases in TMRM labeling observed with the purified proteins upon addition of TDG are on average $\sim 60 \%$ of those observed with RSO membrane vesicles. However, this is not surprising since it is known that a lipid bilayer (le Coutre et al. 1997) as well as its
TDG (-TDG; blue diamond) or preincubated for 10 min with TDG prior to addition of TMRM (+TDG; pink square). Relative TMRM labeling rates were calculated as described (Nie and Kaback 2010); the data are plotted relative to the 20 -s points in the absence (top) or presence (bottom) of TDG. For SDS/PAGE gels, upper gel displays the results of TMRM labeling and bottom is the silver-stained protein sample (Color figure online)

composition (Bogdanov et al. 2002) are important constraints on the structure of LacY.

\section{Single-Molecule Fluorescence (Förster) Resonance Energy Transfer}

Single-molecule fluorescence (Förster) resonance energy transfer (sm-FRET) has also been used to test the alternating access model with wild-type LacY and mutant 
Table 1 TDG changes the rate of TMRM labeling of single-Cys mutants

\begin{tabular}{llcc}
\hline LacY mutant & Helix & $\begin{array}{l}\text { Fold change in TMRM } \\
\text { labeling in RSO vesicles }\end{array}$ & $\begin{array}{c}\text { Fold change in TMRM } \\
\text { labeling in DDM }\end{array}$ \\
\hline $\begin{array}{l}\text { Periplasmic } \\
\text { P31C }\end{array}$ & I & 13.6 & 8.9 \\
K42C & II & 8.5 & 5.9 \\
D44C & II & 8.2 & 6.2 \\
Q242C & II & 5.2 & 3.2 \\
N245C & VII & 14.9 & 6.9 \\
Cytoplasmic & & & -3.8 \\
Q60C & II & -7.2 & -8.3 \\
S67C & II & -14.7 & -3.9 \\
A279C & IX & -13.1 & -3.0 \\
L329C & X & -2.6 & -4.3 \\
V331C & X & -5.1 & \\
\hline
\end{tabular}

Rates of TMRM labeling were obtained from the time courses shown in Fig. 3 as described (Nie and Kaback 2010). For each mutant, the ratio of the estimated initial rate of TMRM labeling in the presence of TDG relative to that observed in the absence of TDG was calculated. Positive numbers indicate an increase in the relative labeling rate due to addition of TDG (periplasmic) and negative numbers indicate a decrease in the relative labeling rate due to addition of TDG (cytoplasmic)

Fig. 4 Ligand-induced effects on the FRET distribution $E^{*}$ at the cytoplasmic side of LacY (R73C/S401C, helices III and XII) or at the periplasmic side of LacY (I164C/S375C, helices V and XI). Top LacY backbone with donor (magenta) and acceptor fluorophores on the cytoplasmic or periplasmic side as indicated. Bottom

a Frequency vs. $E^{*}$ histograms corresponding to wild-type (a,

c) and $\mathrm{C} 154 \mathrm{G}$ mutant $(\mathbf{b}$,

d) LacY. Measurements for each construct were obtained in the absence of sugar (gray) and in the presence of $1 \mathrm{mM}$ (saturating) galactosidic sugar concentration (red) or $1 \mathrm{mM}$ glucosidic sugar (blue) (Nie et al. 2007). High $E^{*}$ indicates high smFRET (i.e., closer distance between the fluorophores); low $E^{*}$ indicates low smFRET (i.e., further distance) (Color figure online)
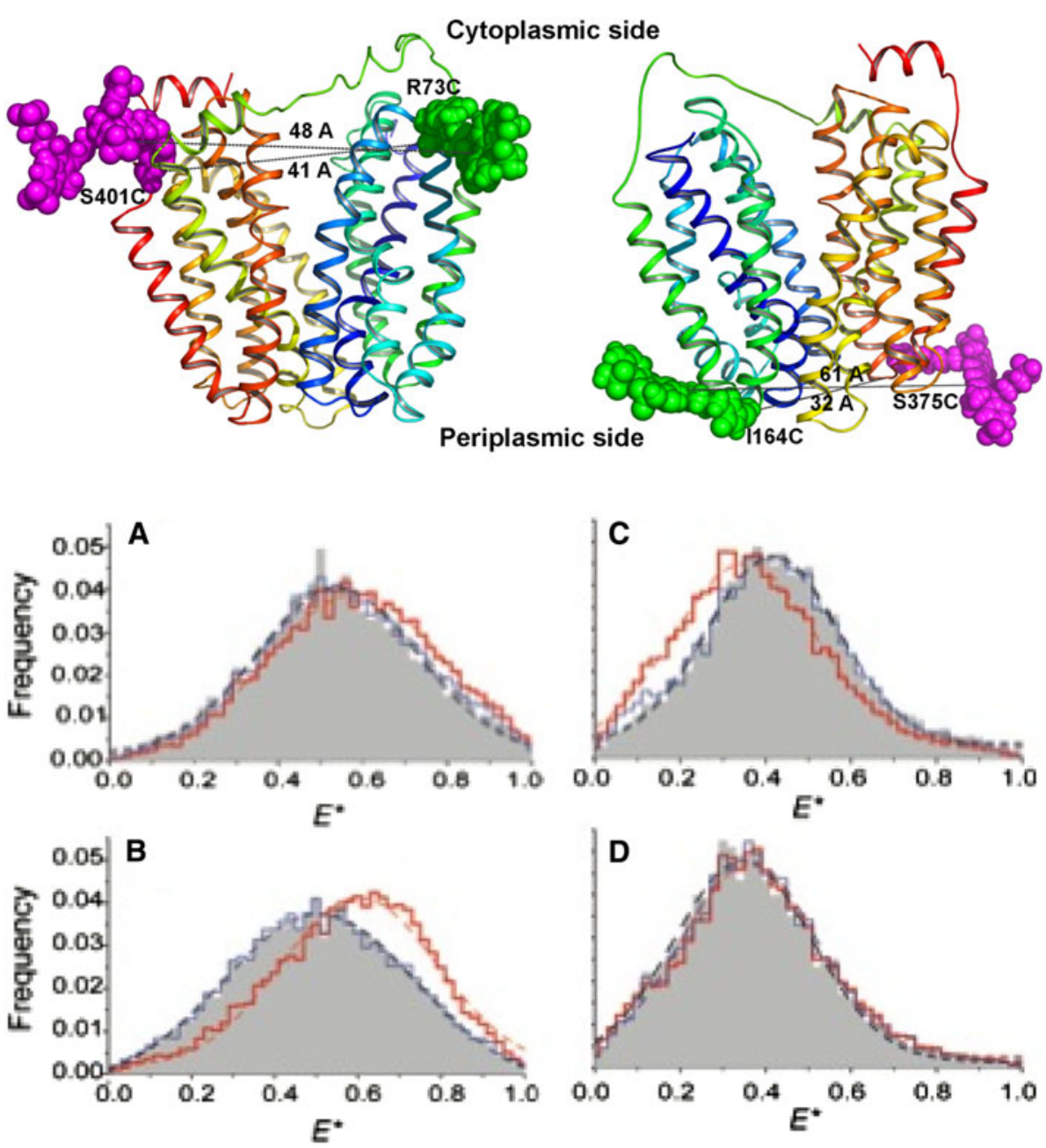
Fig. 5 Top Disulfide-linked nitroxide chains are modeled on the LacY X-ray structure (PDB ID 1PV6, rainbow-colored ribbons from blue [helix I] to red [helix XII]) with the cavity open to the cytoplasmic side.

Nitroxides attached to the backbone of the protein are shown as balls and sticks. Interspin distances used for measurements are shown as dashed lines. Cytoplasmic pairs are viewed from the side (a) and from cytoplasm (b). Periplasmic pairs are viewed from the side (a) and from periplasm (c). Structure of the nitroxide side chain attached to the protein with indicated dihedral angles (d). Bottom DEER

characterization of sugarbinding effects on interspin distances of nitroxide-labeled double-cysteine mutants located on the cytoplasmic (e) or the periplasmic (f) side of LacY. Distance distributions obtained by Tikhonov regularization are shown for protein with no sugar bound (glucosidic sugars sucrose or NPGlc-green or blue, respectively) and with bound sugar (galatosidic sugars TDG or NPGal—pink or red lines, respectively), multigaussian fits (black lines) demonstrating relative distributions of conformational populations. See Smirnova et al. (2007) for details (Color figure online)
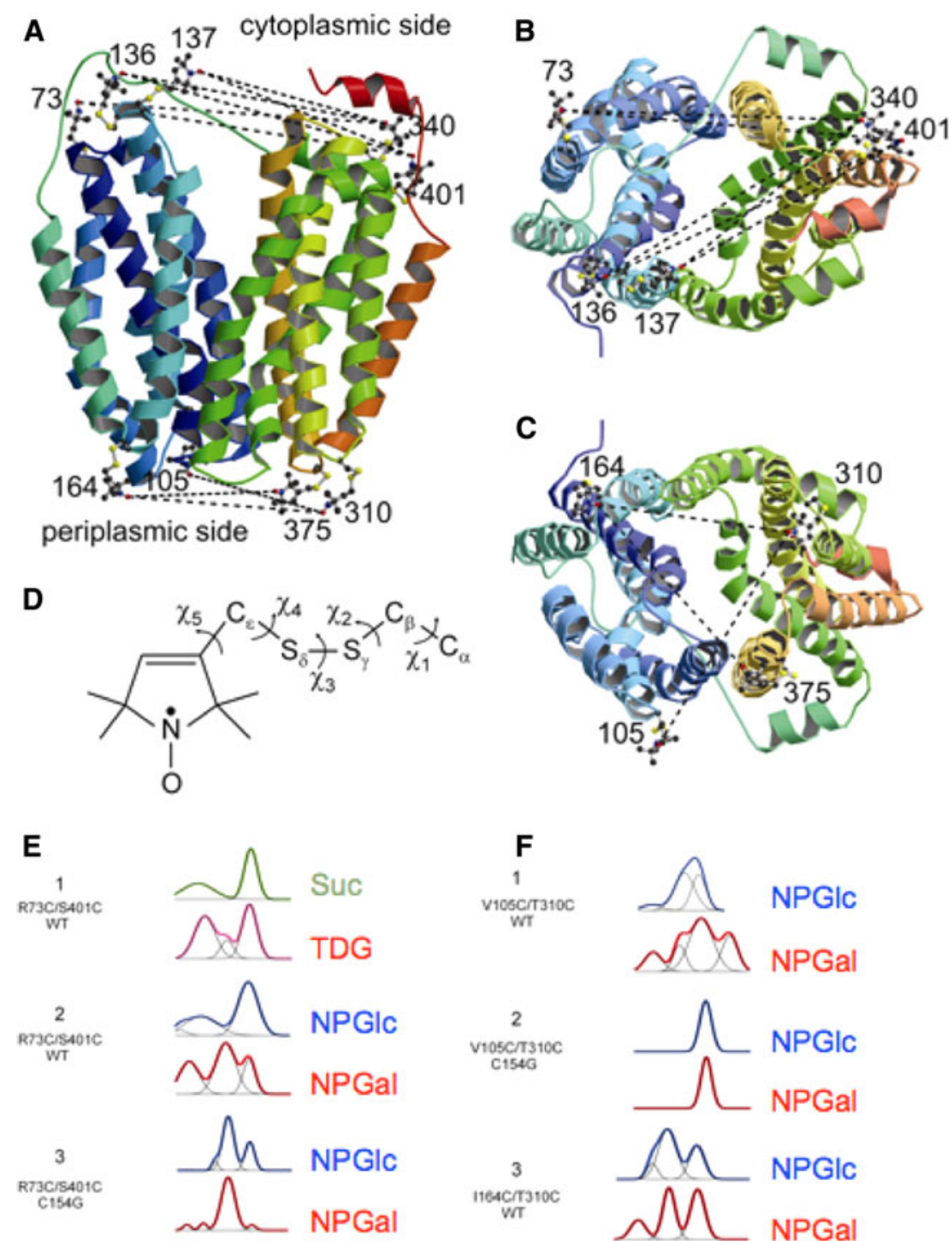

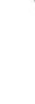

R73C/S401C
C154G
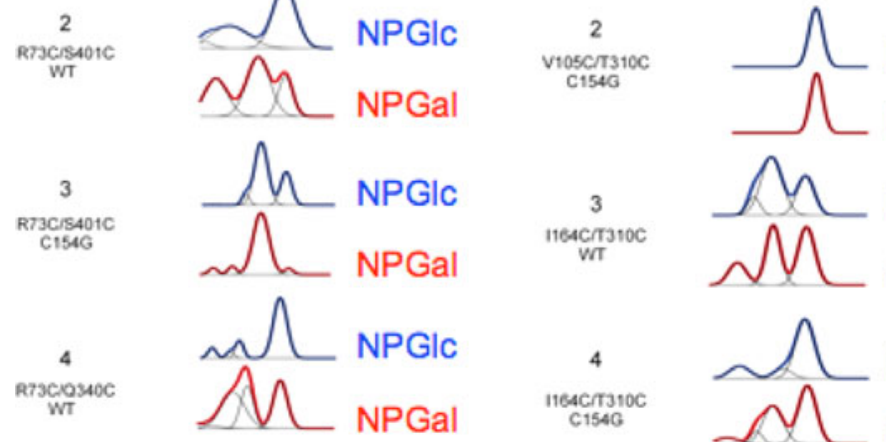

NPGIC

NPGal

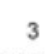

$1164 \mathrm{C} / T 310 \mathrm{C}$
VTT

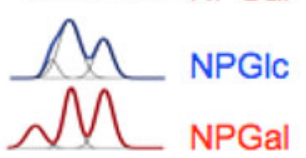

4


NPGIC

C154G

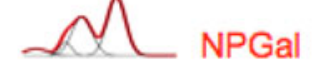

5


NPGIC

NPGal

Inter-spin distance $\AA$

C154G in collaboration with Devdoot Majumdar and Shimon Weiss (Majumdar et al. 2007). Pairs of Cys residues at the ends of two helices on the cytoplasmic or periplasmic side of wild-type LacY and the mutant were labeled with appropriate donor and acceptor fluorophores, sm-FRET was determined in the absence and presence of sugar and distance changes were calculated. With wildtype LacY, binding of a galactopyranoside, but not a glucopyranoside, results in a decrease in distance on the cytoplasmic side and an increase in distance and in distance distribution on the periplasmic side (Fig. 4). In contrast, with the mutant, more pronounced decreases in distance and in distance distribution are observed on the cytoplasmic side but there is no change on the periplasmic side (Fig. 4). The results are consistent with the alternating access model and indicate that the translocation defect in the mutant is due to paralysis in the outward-facing conformation. 


\section{Double Electron-Electron Resonance}

Double electron-electron resonance (DEER), a site-directed spin labeling technique, was applied to measure interhelical distance changes induced by sugar binding in collaboration with Christian Altenbach and Wayne Hubbell (Smirnova et al. 2007). Nitroxide-labeled paired-Cys replacements were constructed at the ends of transmembrane helices on the cytoplasmic or periplasmic side of LacY and in the conformationally restricted mutant C154G (Fig. 5a-d). Distances were then determined in the presence of galactosidic or nongalactosidic sugars (Fig. 5e, f). Strikingly, specific binding causes conformational rearrangements on both sides of the molecule. On the cytoplasmic side, each of six nitroxide-labeled pairs exhibits decreased interspin distances, ranging 4-21 A. Conversely, on the periplasmic side, each of three spin-labeled pairs shows increased distances, ranging 4-14 ̊. Thus, the inward-facing cytoplasmic cavity closes and a cavity opens on the tightly packed periplasmic side. In the $\mathrm{C} 154 \mathrm{G}$ mutant, sugar-induced closing is observed on the cytoplasmic face but little or no change occurs on periplasmic side. DEER measurements in conjunction with molecular modeling based on the X-ray structure provide strong support for the alternative access model and suggest a structure for the outward-facing conformation of LacY.

\section{Site-Directed Cross-Linking}

As discussed, the residues essential for sugar recognition and $\mathrm{H}^{+}$translocation are located at the apex of the cavity and are inaccessible from the outside. On the periplasmic side, helices I/II and VII from the N and C six-helix bundles, respectively, participate in sealing the cavity from the outside. Three paired double-Cys mutants-Ile40 $\rightarrow$ Cys/ Asn245 $\rightarrow$ Cys, Thr45 $\rightarrow$ Cys/Asn245 $\rightarrow$ Cys and Ile32 $\rightarrow$ Cys/Asn245 $\rightarrow$ Cys-located in the interface between helices I/II and VII on the periplasmic side of $\mathrm{LacY}$ were constructed with tandem factor $\mathrm{Xa}$ protease

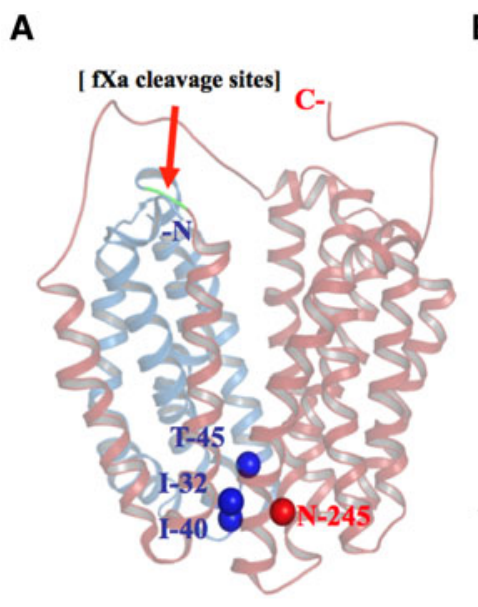

C

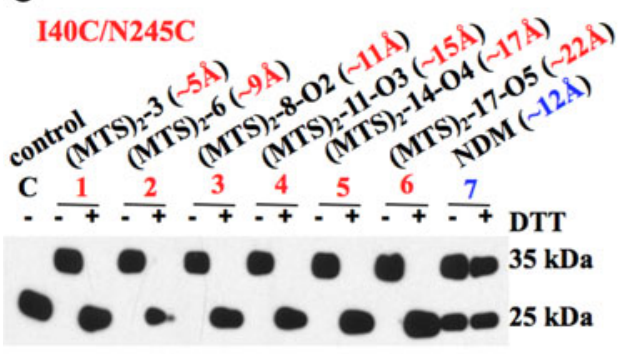

Fig. 6 a Structure model of LacY (PDB ID 2V8 N). Residues Ile32 (helix I), Ile40 (loop I/II) and Thr45 (helix II) are presented as dark blue spheres, and residue $\mathrm{N} 245$ (helix VII) is presented as a red sphere. The $\mathrm{N}$-terminal four helices $\left(\mathrm{N}_{4}\right)$ and the $\mathrm{C}$-terminal helices $\left(\mathrm{C}_{8}\right)$ are shown in blue and red, respectively, and separated by tandem factor Xa protease sites (left side, green), as indicated by the red arrow. b Homobifunctional cross-linking reagents. For MTS reagents,

7. NDM
B Cross-linkers

1. MTS-3-MTS

2. MTS-6-MTS

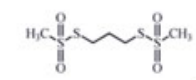

Distance (approximate)

3. MTS-8-O2-MTS

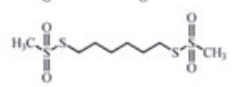

$\sim 5 \AA$

$\rightarrow \mathbf{A}$

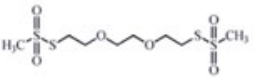

$\sim 11 \AA$

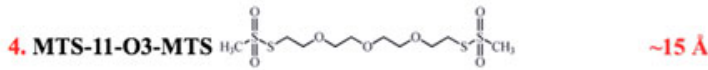

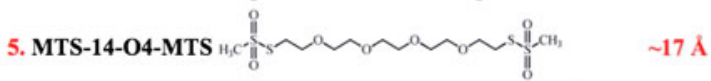

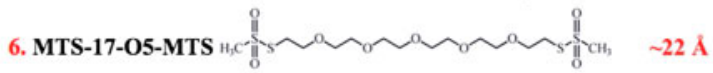
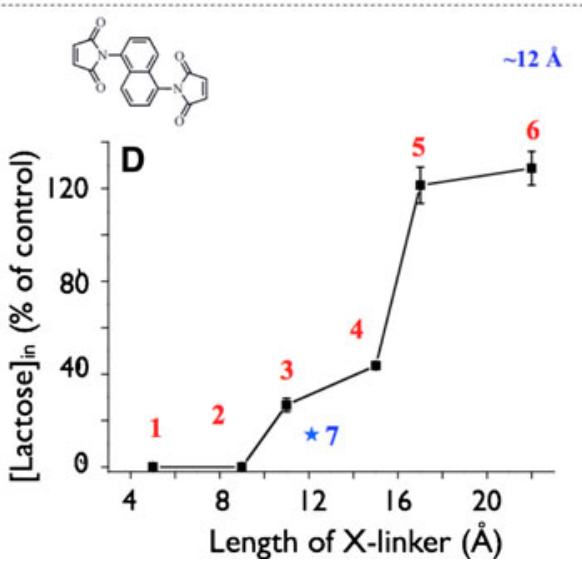

the approximate $\mathrm{S}-\mathrm{S}$ distances between bridging sulfur atoms in the chains are as given by the manufacturer. The distance for NDM is from Green et al. (2001). Cross-linking (c) and lactose transport (d) with mutant I40C/N245C. All experiments were performed with RSO vesicles. 1, MTS-3-MTS; 2, MTS-6-MTS; 3, MTS-8-O2-MTS; 4, MTS-11-O3-MTS; 5, MTS-14-O4-MTS; 6, MTS-17-O5-MTS; 7, NDM (Color figure online) 
sites between the two Cys replacements (Fig. 6a) (Zhou et al. 2008). After quantitative cross-linking with flexible homo-bifunctional reagents less than about $15 \AA$ in length, all three mutants lose the ability to catalyze lactose transport (Fig. 6b-d). Strikingly, however, full or partial activity is observed when cross-linking is mediated by flexible reagents greater than about $15 \AA$ in length. Moreover, $17 \AA$ is the minimum required for maximum activity, a distance very similar to that obtained from DEER (Smirnova et al. 2007). The results provide further support for the argument that transport via LacY involves opening and closing of a large periplasmic cavity.

\section{Trp Quenching}

Since Trp fluorescence is quenched by certain amino acyl side chains such as a protonated His or amino group, Trp residues were placed on either side of LacY where they are predicted to be in close proximity to the imidazole side chains of His in either the inward- or outward-facing conformation (Fig. 7, top) (Smirnova et al. 2009a). In the inward-facing conformation, LacY is tightly packed on the periplasmic side and Trp residues placed at position 245 (helix VII) or 378 (helix XII) are in close contact with His35 (helix I) or Lys42 (helix II), respectively. Sugar binding leads to unquenching of Trp fluorescence in both mutants, a finding clearly consistent with opening of the periplasmic cavity (Fig. 7a). The $\mathrm{pH}$ dependence of Trp245 unquenching exhibits a $\mathrm{p} K \mathrm{a}$ of $\sim 8$, typical for a His side chain interacting with an aromatic group. On the cytoplasmic side, Phe140 (helix V) and Phe334 (helix X) are located on opposite sides of a wide open hydrophilic cavity. In precisely the opposite fashion from the periplasmic side, mutant Phe140 $\rightarrow$ Trp/Phe334 $\rightarrow$ His exhibits sugar-induced Trp quenching (Fig. 7b). Again,

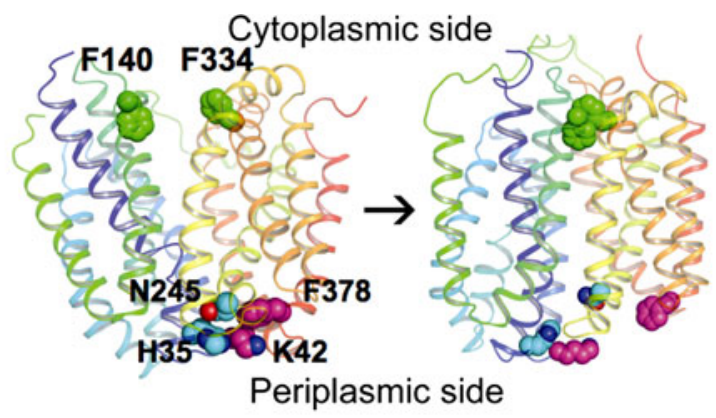

\section{Cytoplasmic pair \\ $\mathrm{F} 140 \mathrm{~W}-\mathrm{F} 334 \mathrm{H}$ \\ $\mathrm{HV}-\mathrm{HX}$ \\ Periplasmic pairs \\ $\mathrm{N} 245 \mathrm{~W}-\mathrm{H} 35$ \\ $\mathrm{HVII}-\mathrm{HI}$ \\ $\mathrm{F} 378 \mathrm{~W}-\mathrm{K} 42$ \\ HXII - HII}

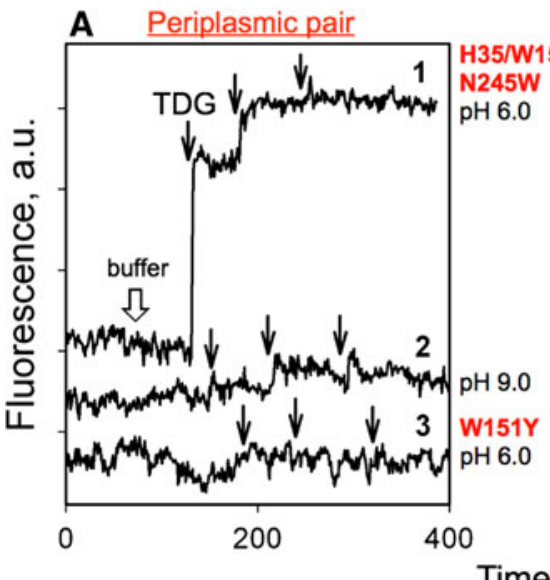

Time (s)

Fig. 7 Top Pairs of amino acid residues selected for Trp substitutions are shown on the backbone structure of LacY. The F140-F334 pair (helices $\mathrm{V}$ and $\mathrm{X}$ ) on the cytoplasmic side is shown as green spheres. The H35-N245 (helices I and VII) and K42-F378 (helices II and XII) pairs on the periplasmic side are shown as cyan and pink spheres, respectively. A side view of the overall structure in the inward-facing conformation (PDB ID 2CFQ) is shown on the left, and the structure in the outward-facing conformation modeled (Smirnova et al. 2007) is shown on the right. Transmembrane helices are rainbow-colored from blue (helix I) to red (helix XII). Arrow indicates the conformational

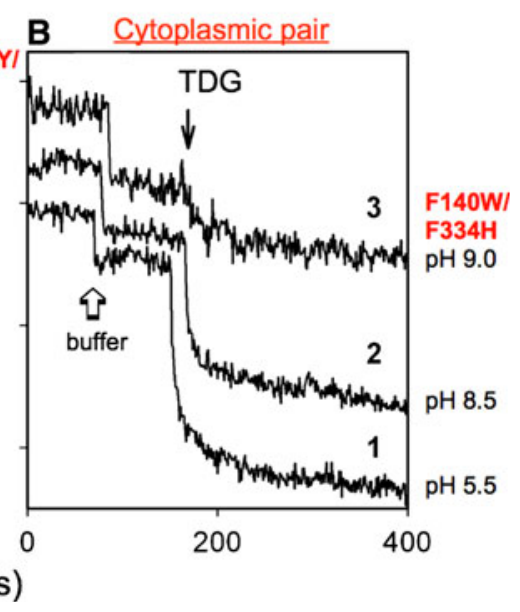

change resulting from sugar binding. The LacY structure is presented using Pymol 0.97 (DeLano Scientific, San Carlos, CA). a TDG effect on fluorescence of Trp introduced on the periplasmic side. Sequential additions of $6 \mu \mathrm{l}$ of buffer (open arrow) and 6- $\mu$ l aliquots of 1.8 M TDG (black arrows) to the mutant W151Y/N245 W at pH 6.0 (trace 1) or at $\mathrm{pH} 9.0$ (trace 2) and to the control mutant $\mathrm{W} 151 \mathrm{Y}$ at $\mathrm{pH} 6.0$ (trace 3). b TDG effect on Trp fluorescence of cytoplasmic pair F140 W-F334H at pH 5.5 (trace 1), $\mathrm{pH} 8.5$ (trace 2) and $\mathrm{pH} 9.0$ (trace 3). Additions of $20 \mu \mathrm{l}$ of buffer (open arrows) or $20 \mu \mathrm{l}$ of $1.5 \mathrm{M}$ TDG (black arrows) were made into $2 \mathrm{ml}$ of protein solutions (Color figure online) 
quenching is $\mathrm{pH}$-dependent with a $\mathrm{pKa}$ of $\sim 8$. The results provide yet another strong, independent line of evidence for the alternating access mechanism and demonstrate that the methodology described provides a sensitive probe to measure conformational changes in membrane transport proteins.

Acknowledgements The authors are indebted to M. Gregor Madej for embellishing the graphics and proofreading the manuscript. This work was supported by NIH grants DK051131, DK069463, GM073210 and GM074929 and NSF grant 0450970 (to H. R. K.).

Open Access This article is distributed under the terms of the Creative Commons Attribution Noncommercial License which permits any noncommercial use, distribution, and reproduction in any medium, provided the original author(s) and source are credited.

\section{References}

Abramson J, Smirnova I, Kasho V, Verner G, Kaback HR, Iwata S (2003) Structure and mechanism of the lactose permease of Escherichia coli. Science 301:610-615

Bogdanov M, Heacock PN, Dowhan W (2002) A polytopic membrane protein displays a reversible topology dependent on membrane lipid composition. EMBO J 21:2107-2116

Frillingos S, Kaback HR (1996) Cysteine-scanning mutagenesis of helix VI and the flanking hydrophilic domains in the lactose permease of Escherichia coli. Biochemistry 35:5333-5338

Green NS, Reisler E, Houk KN (2001) Quantitative evaluation of the lengths of homobifunctional protein cross-linking reagents used as molecular rulers. Protein Sci 10:1293-1304

Guan L, Kaback HR (2006) Lessons from lactose permease. Annu Rev Biophys Biomol Struct 35:67-91

Guan L, Kaback HR (2007) Site-directed alkylation of cysteine to test solvent accessibility of membrane proteins. Nat Protoc 2:2012-2017

Guan L, Mirza O, Verner G, Iwata S, Kaback HR (2007) Structural determination of wild-type lactose permease. Proc Natl Acad Sci USA 104:15294-15298

Huang Y, Lemieux MJ, Song J, Auer M, Wang DN (2003) Structure and mechanism of the glycerol-3-phosphate transporter from Escherichia coli. Science 301:616-620

Kaback HR (2005) Structure and mechanism of the lactose permease. C R Biol 328:557-567

Kaback HR, Sahin-Toth M, Weinglass AB (2001) The kamikaze approach to membrane transport. Nat Rev Mol Cell Biol 2:610-620

Kaback HR, Dunten R, Frillingos S, Venkatesan P, Kwaw I, Zhang W, Ermolova N (2007) Site-directed alkylation and the alternating access model for LacY. Proc Natl Acad Sci USA 104:491-494

le Coutre J, Narasimhan LR, Patel CK, Kaback HR (1997) The lipid bilayer determines helical tilt angle and function in lactose permease of Escherichia coli. Proc Natl Acad Sci USA 94:10167-10171

le Coutre J, Kaback HR, Patel CK, Heginbotham L, Miller C (1998) Fourier transform infrared spectroscopy reveals a rigid alphahelical assembly for the tetrameric Streptomyces lividans $\mathrm{K}^{+}$ channel. Proc Natl Acad Sci USA 95:6114-6117
Liu Z, Madej MG, Kaback HR (2010) Helix dynamics in LacY: helices II and IV. J Mol Biol 396:617-626

Majumdar DS, Smirnova I, Kasho V, Nir E, Kong X, Weiss S, Kaback HR (2007) Single-molecule FRET reveals sugar-induced conformational dynamics in LacY. Proc Natl Acad Sci USA 104:12640-12645

Mirza O, Guan L, Verner G, Iwata S, Kaback HR (2006) Structural evidence for induced fit and a mechanism for sugar $/ \mathrm{H}^{+}$symport in LacY. EMBO J 25:1177-1183

Nie Y, Kaback HR (2010) Sugar binding induces the same global conformational change in purified LacY as in the native bacterial membrane. Proc Natl Acad Sci USA 107:9903-9908

Nie Y, Smirnova I, Kasho V, Kaback HR (2006) Energetics of ligandinduced conformational flexibility in the lactose permease of Escherichia coli. J Biol Chem 281:35779-35784

Nie Y, Ermolova N, Kaback HR (2007) Site-directed alkylation of LacY: effect of the proton electrochemical gradient. J Mol Biol 374:356-364

Nie Y, Sabetfard FE, Kaback HR (2008) The Cys154 $\rightarrow$ Gly mutation in LacY causes constitutive opening of the hydrophilic periplasmic pathway. J Mol Biol 379:695-703

Nie Y, Zhou Y, Kaback HR (2009) Clogging the periplasmic pathway in LacY. Biochemistry 48:738-743

Patzlaff JS, Moeller JA, Barry BA, Brooker RJ (1998) Fourier transform infrared analysis of purified lactose permease: a monodisperse lactose permease preparation is stably folded, alpha-helical, and highly accessible to deuterium exchange. Biochemistry 37:15363-15375

Sayeed WM, Baenziger JE (2009) Structural characterization of the osmosensor ProP. Biochim Biophys Acta 1788:1108-1115

Smirnova I, Kasho V, Choe JY, Altenbach C, Hubbell WL, Kaback HR (2007) Sugar binding induces an outward facing conformation of LacY. Proc Natl Acad Sci USA 104:16504-16509

Smirnova I, Kasho V, Sugihara J, Kaback HR (2009a) Probing of the rates of alternating access in $\mathrm{LacY}$ with Trp fluorescence. Proc Natl Acad Sci USA 106:21561-21566

Smirnova IN, Kasho VN, Sugihara J, Choe JY, Kaback HR (2009b) Residues in the $\mathrm{H}^{+}$translocation site define the $\mathrm{pKa}$ for sugar binding to LacY. Biochemistry 48:8852-8860

van Iwaarden PR, Driessen AJ, Menick DR, Kaback HR, Konings WN (1991) Characterization of purified, reconstituted sitedirected cysteine mutants of the lactose permease of Escherichia coli. J Biol Chem 266:15688-15692

Venkatesan P, Kaback HR (1998) The substrate-binding site in the lactose permease of Escherichia coli. Proc Natl Acad Sci USA 95:9802-9807

Venkatesan P, Kwaw I, Hu Y, Kaback HR (2000a) Site-directed sulfhydryl labeling of the lactose permease of Escherichia coli: helix VII. Biochemistry 39:10641-10648

Venkatesan P, Liu Z, Hu Y, Kaback HR (2000b) Site-directed sulfhydryl labeling of the lactose permease of Escherichia coli: helix II. Biochemistry 39:10649-10655

Viitanen P, Newman MJ, Foster DL, Wilson TH, Kaback HR (1986) Purification, reconstitution, and characterization of the lac permease of Escherichia coli. Methods Enzymol 125:429-452

Zhou Y, Guan L, Freites JA, Kaback HR (2008) Opening and closing of the periplasmic gate in lactose permease. Proc Natl Acad Sci USA 105:3774-3778

Zhou Y, Nie Y, Kaback HR (2009) Residues gating the periplasmic pathway of LacY. J Mol Biol 394:219-225 